\title{
Optical microscopic study of surface morphology and filtering efficiency of face masks
}

\author{
Bhanu Bhakta Neupane ${ }^{\text {Corresp., } 1}$, Sangeeta Mainali ${ }^{2}$, Amita Sharma $^{3}$, Basant Giri $^{3}$ \\ ${ }^{1}$ Central Department of Chemistry, Tribhuvan University, Kathamndu, Nepal \\ 2 Department of Chemistry, Amrit Campus, Tribhuvan University, Kathmanu, Nepal \\ 3 Center for Analytical Sciences, Kathmandu Institute of Applied Sciences, Kathmandu, Nepal \\ Corresponding Author: Bhanu Bhakta Neupane \\ Email address: bbneupane@cdctu.edu.np
}

Background. Low cost face masks made from different cloth materials are very common in developing countries. The cloth masks are usually double layered with stretchable ear loops. It is common practice to use such masks for months after multiple washing and drying cycles. If a cloth mask is used for long time, ear loops get stretched. The loop requires to be knotted to make the mask loop fit better on the face. It is not clear how washing and drying and stretching practices change the quality of a cloth mask.

Particulate matter filtering efficiency of a mask depends on multiple parameters, such as pore size, shape, clearance, and pore number density. It is important to understand the effect of these parameters on the filtering efficiency. Methods. We characterized the surface of twenty different types of cloth masks using optical image analysis method. The filtering efficiency of selected cloth face masks was measured using particle counting method. We also studied the effect of washing and drying and stretching on quality of a mask. Results. The pore size of masks ranged from 80-500 micrometer which was much bigger than particular matter having diameter of $2.5 \mu \mathrm{m}$ or less $\left(\mathrm{PM}_{2.5}\right)$ and $10 \mu \mathrm{m}$ or less $\left(\mathrm{PM}_{10}\right)$ size. The $\mathrm{PM}_{10}$ filtering efficiency of four of the selected masks ranged from $63-84 \%$. The poor filtering efficiency may have arisen from larger and open pores present in the masks. Interestingly, we found that after $4^{\text {th }}$ washing and drying cycle efficiency dropped by $20 \%$. We observed change in pore size and shape and decrease in microfibers within the pores after washing. Stretching of cloth mask surface also altered the pore size and potentially decreased the filtering efficiency. As compared to cloth masks, the less frequently used surgical/paper masks had complicated networks of fibers and much smaller pores in multiple layers in comparison to cloth masks, therefore had better filtering efficiency. This study showed that filtering efficiency of cloth face mask was relatively lower and washing and drying practices deteriorated the efficiency. We believe that the 
findings of this study will be very helpful to increase public awareness and help governmental agencies to make proper guidelines and policies for use of face mask. 
1 2

${ }^{1}$ Central Department of Chemistry, Tribhuvan University, Kathmandu, Nepal

$4 \quad{ }^{2}$ Department of Chemistry, Amrit Campus, Tribhuvan University, Kathmandu, Nepal

$5 \quad{ }^{3}$ Center for Analytical Sciences, Kathmandu Institute of Applied Sciences, Kathmandu, Nepal

6

7

8

9

10

11

12

13

14

15

16

17

18

19

20

21

22

23

24

25

26

27

28
*Corresponding author

Bhanu Bhakta Neupane

Central Department of Chemistry, Tribhuvan University, Kathmandu, Nepal

Email: bbneupane@cdctu.edu.np

\section{Optical microscopic study of surface morphology and filtering efficiency of face masks}

\author{
Bhanu Bhakta Neupane ${ }^{1 *}$, Sangeeta Mainali², Amita Sharma ${ }^{3}$, Basant Giri ${ }^{3}$
}

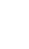

14


30

Background. Low cost face masks made from different cloth materials are very common in developing countries. The cloth masks are usually double layered with stretchable ear loops. It is common practice to use such masks for months after multiple washing and drying cycles. If a cloth mask is used for long time, ear loops get stretched. The loop requires to be knotted to make the mask loop fit better on the face. It is not clear how washing and drying and stretching practices change the quality of a cloth mask. Particulate matter filtering efficiency of a mask depends on multiple parameters, such as pore size, shape, clearance, and pore number density. It is important to understand the effect of these parameters on the filtering efficiency.

Methods. We characterized the surface of twenty different types of cloth masks using optical image analysis method. The filtering efficiency of selected cloth face masks was measured using particle counting method. We also studied the effect of washing and drying and stretching on quality of a mask.

Results. The pore size of masks ranged from 80-500 micrometer which was much bigger than particular matter having diameter of $2.5 \mu \mathrm{m}$ or less $\left(\mathrm{PM}_{2.5}\right)$ and $10 \mu \mathrm{m}$ or less $\left(\mathrm{PM}_{10}\right)$ size. The $\mathrm{PM}_{10}$ filtering efficiency of four of the selected masks ranged from $63-84 \%$. The poor filtering efficiency may have arisen from larger and open pores present in the masks. Interestingly, we found that after $4^{\text {th }}$ washing and drying cycle efficiency dropped by $20 \%$. We observed change in pore size and shape and decrease in microfibers within the pores after washing. Stretching of cloth mask surface also altered the pore size and potentially decreased the filtering efficiency. As compared to cloth masks, the less frequently used surgical/paper masks had complicated networks of fibers and much smaller pores in multiple layers in comparison to cloth masks, therefore had better filtering efficiency.

This study showed that filtering efficiency of cloth face mask was relatively lower and washing and drying practices deteriorated the efficiency. We believe that the findings of this study will be very helpful to increase public awareness and help governmental agencies to make proper guidelines and policies for use of face mask. 


\section{INTRODUCTION}

Particulate matter (PM) contributes significantly to overall ambient air pollution. The microscopic particles having diameter of $2.5 \mu \mathrm{m}$ or less $\left(\mathrm{PM}_{2.5}\right)$ can deposit to the conducting zone of the respiratory system including the alveoli and penetrate to the cardiovascular system. These fine particles are believed to be responsible for various health problems such as lung inflammation and lung cancer, vascular dysfunction, myocardial infarction, and exacerbation of existing conditions like asthma, diabetes mellitus (Reche et al., 2012; Kim, Kabir \& Kabir, 2015; World Health Organization, 2016). The World Health Organization (WHO) estimates that ambient air pollution caused around 4.2 million deaths in 2016, with Asia alone contributing around $60 \%$ of total global burden(World Health Organization, 2016).

The PM pollution in many cities globally is higher than the WHO recommended safe limit(Wang et al., 2008; Colbeck, Nasir \& Ali, 2010; Sharma et al., 2014; World Health Organization, 2016; Maleki et al., 2016). For example, in Kathmandu valley, one of the most densely populated and the fastest-growing cities in Asia, air quality is very poor with PM alone contributing to around $50-60 \%$ of total pollution burden. The ambient annual average $\mathrm{PM}_{2.5}$ concentration levels in core urban areas of Kathmandu, Nepal has been reported to be around $54 \mu \mathrm{g} / \mathrm{m}^{3}$ which is significantly higher than the WHO standard of $10 \mu \mathrm{g} / \mathrm{m}^{3}$ (World Health Organization, 2016; IQ Air, 2018). In recent year, a systematic study on exposure of $\mathrm{PM}_{2.5}$ (finer fractions of $\mathrm{PM}_{10}$ ) to subjects of different occupations was made in few core and sub-urban areas of Kathmandu valley. The hourly personal $\mathrm{PM}_{2.5}$ exposure to traffic police who spend 8-12 hours on road duty was found in the range of $40-80 \mu \mathrm{g} / \mathrm{m}^{3}$ (Gurung \& Bell, 2012), which is $1.6-3.2$ times higher than WHO standard for twenty four hours average of $25 \mu \mathrm{g} / \mathrm{m}^{3}$ (World Health Organization, 2016). 
82 The PM pollution can be minimized by put forwarding both short and long terms policies and

83 regulations and enforcing them. Efforts have been made but their impact in minimizing pollution

84 is almost futile in cities of developing countries.

85 Studies have shown that face masks reduce the exposure to particulate matter and other

86 contaminants (Singh et al., 2010; Chughtai, Seale \& MacIntyre, 2013; MacIntyre et al., 2015;

87 Shakya et al., 2017). Most commonly used face masks are cloth masks (CM) and surgical masks

88 (SM). The effectiveness of a mask is measured by its filtering efficiency. A mask is considered

89 effective if its filtering efficiency is greater than 95\%. Efficacy of a mask depends on type of mask

90 material (Mueller et al., 2018), particle size and charge of aerosol tested (Shakya et al., 2017), and

91 user preference (Maxted, 2011; Chughtai, Seale \& MacIntyre, 2013).

92 The cloth masks are particularly popular in developing countries because they are inexpensive

93 (unit retail price range: USD 0.08-1.5), locally available, and reusable after washing. The cloth

94 masks are usually double layered (2 ply masks) with stretchable ear loops (see Fig. 1A and B).

95 The face masks have also been widely used in healthcare facilities to minimize

96 contamination(Chughtai, Seale \& MacIntyre, 2013), in emergency situations such as volcanic

97 eruptions(Mueller et al., 2018) and in occupational hazard protection(Belkin, 1997; van der Sande,

98 Teunis \& Sabel, 2008; MacIntyre \& Chughtai, 2015; Cherrie et al., 2018).

99 Previous studies have reported the measurement of filtering efficiency of cloth masks using lab 100 generated aerosol, polystyrene latex, virus mono-size particles and diesel exhaust (Rengasamy, 101 Eimer \& Shaffer, 2010; MacIntyre et al., 2015; Shakya et al., 2017). As the ambient aerosol we 102 are exposed to a mixture of particles of varying size and shape in ambient environmental condition, 103 use of simulated particles in controlled laboratory setting may not truly represent the filtering 104 efficiency of masks. 
105 Most commonly used face masks in low-income countries are cloth masks. Such masks are used

106 for many days and are also used after washing and drying multiple times. In addition, the one-for-

107 all type ear loop of mask does not fit to everyone. Therefore, the loop has to be knotted or stretched

108 for better fitting on the face. The washing, drying and stretching practices may change the pore

109 size and porosity of the fabric and thus may deteriorate filtering efficiency. There are no published

110 reports on the surface morphology of face masks and the effect of washing and stretching on pore

111 size of the masks.

112 In this work, we report on the detail study on surface characterization (pore size, shape, clearance,

113 and pore distribution) of commonly available cloth face masks purchased from local market of

114 Kathmandu, Nepal and compared with surgical face masks using bright field microscope. We then

115 measured the efficacy of masks on filtering ambient outdoor aerosol particles by particle counting

116 method. Finally we report on the effect of washing and drying cycles and stretching on surface

117 characteristics and filtering efficiency of cloth masks.

118

119 MATERIALS AND METHODS

120 Survey study: We began our study by conducting a survey to know the type of masks people use

121 in Kathmandu. We counted 1500 people walking through the junction road in Kalanki, Kathmandu

122 and noted number of people wearing face mask and type of mask. The visual counting was carried

123 out from 9AM to 1PM for three consecutive days in the month of May 2016. Kalanki is a major

124 traffic intersection in Kathmandu.

125 Surface characterization of masks: Twenty different types of cloth face masks (CM) and seven

126 different brands of surgical masks (SMs) were purchased from local markets in Kathmandu (Fig.

127 1A and B). The cloth mask types were selected on the basis of design and fabric material. A small 
128 section of each mask was cut and was imaged using an optical microscope in bright field mode 129 using objective of 0.4 NA (10X, air) with total magnification of 100X. A built in white LED was 130 used as illumination source and image was acquired by a CMOS camera (AmScope, USA). For 131 each mask ten images were taken and the collected images were processed in ImageJ software 132 (NIH, USA). The field of view of measured image was calibrated by using a calibration glass slide 133 having grid size of $10 \mu \mathrm{m}$ (AmScope, USA). The intensity of light used before the lens was around $1341 \mathrm{~mW} / \mathrm{cm}^{2}$. Acquisition time of image, unless specified, was set to 500 milli seconds.

Measurement of filtering efficiency: Microscope coverslips (Corning, 1.5, 22×22 mm) were placed on a polystyrene petri dish (Microteknic, $80 \mathrm{~mm}$ diameter and $13 \mathrm{~mm}$ depth) and the petri dish was covered with a face mask. To make sure coverslip surface is free from particles, the surface was cleaned and inspected by the bright field microscope. To ensure no marginal leakage of particles, the mask was fastened to the petri dish with a rubber band. The whole assembly was kept at twenty feet above the ground in an open box in central Kathmandu. Major sources of particulate matter pollution in this area are vehicular exhausts, dust suspensions, and particles from poorly maintained roads. For control measurement, a second assembly was made without mask and kept in the same place. Both assemblies were exposed to particulate matter for 30 minutes and number of particles deposited on the glass coverslip surface was counted after the surface was imaged at magnification of $100 \mathrm{X}$ with a bright field microscope.

146 One mask type was sampled at a time and triplicate measurements were carried out for each mask.

147 All experiments were made in sunny days during 11:00AM to 1:00PM time period. Although we 148 did not measure the wind velocity at our site, the wind velocity was low $(\sim 10 \mathrm{~km} / \mathrm{h})$ at Tribhuvan 149 International Airport which is just $2 \mathrm{~km}$ aerial distance away.

150 The filtering efficiency was calculated as: 
151 Filtering efficiency $(\%)=\frac{\text { number of particles with mask }}{\text { number of particles without mask }} \times 100$

152 To measure the mask efficiency after washing and drying cycles, mask was soaked for one hour

153 in $2 \%(\mathrm{w} / \mathrm{v})$ aqueous solution of powder detergent that contained alkyl-benzene sulfonate and

154 sodium triphosphate as main ingredients. The mask was rinsed multiple times with water so as to

155 get rid of the detergent. The mask was then laid on a flat surface to make sure no stretching of the

156 cloth fibers, and the mask was air dried. Filtering efficiency was measured after each washing and

157 drying cycle by using the procedure mentioned in above paragraph.

158 To the best of our knowledge, the particle counting method stated above is novel for the 159 determination of filtering efficiency of a mask.

160 Determination of particle size: To measure the particle size, particles were deposited on coverslip 161 and imaged at $100 \mathrm{X}$ magnification using the bright field microscope. The resolution of the

162 microscopic system was 0.6 micrometer. The field of view of measured image was calibrated by 163 using a calibration glass slide having grid size of $10 \mu \mathrm{m}$ (AmScope, USA), and particles size was 164 estimated.

\section{RESULTS}

\section{Survey study}

168 A survey conducted in Kalanki, Kathmandu, Nepal showed that 31\% people used face mask while they were at or close to busy roads (see Fig. 1C). Kalanki is an urban area of Kathmandu and a major cross-section for exit and entry to the Kathmandu valley from rest of the Nepal. It gets large number of vehicular movements since early morning to late night. 
Figure 1. Types of mask and survey. (A) Images of some of the most commonly used cloth masks, and less commonly used surgical mask (B). (C) Face mask use pattern in Kathmandu. The \% errors in the Fig. represent standard deviation.

\section{Surface characterization}

The representative microscopic images of different cloth masks (CMs) and surgical masks (SM) are shown in Fig. 2. Out of twenty cloth masks (CM1-CM20) imaged, for brevity, images of CM1, CM3, CM7, CM9, CM12, CM18 are shown in A, B, C, D, E, and F, respectively. The bright patches in the image are the pores present in the mask. All CMs studied contained two ply (layers) and the surface characteristics of both layers was very similar.

Figure 2. Bright field microscopic images of mask surfaces. (A) Representative images for CM1, (B) CM3, (C) CM7, (D) CM9, (E) CM12, and (F) CM18. (G) Representative images of inner, (H) middle, and (I) outer layers of a three layered surgical mask. Scale bar shown A is $500 \mu \mathrm{m}$ and applied to all images.

Although the pore shape and size in CMs were not uniform (see Fig. 2A-F), we tried to extract quantitative information on the size of the pores by measuring the longest dimension of each pore. Such measurement provided an upper estimation of the size of a pore in each CM. The mean pore size ranged from 81 to $461 \mu \mathrm{m}$, with smallest pore size observed for CM4 $(81 \pm 29 \mu \mathrm{m})$ and largest pores observed for CM9 $(461 \pm 108 \mu \mathrm{m})$.

Particulate matter filtering efficiency of a mask also depends on number of pores per unit area; referred here to as pore number density. To get this information, we counted number of pores per 
195 microscopic field of view (field of view was $4.5 \mathrm{~mm}^{2}$ ). We found very diverse number of pores

196 ranging from around 12 (CM11) to 47 (CM15).

197 For comparison we also examined the surface of seven different brands of paper/surgical masks

198 (SM) masks available in market. The masks examined contained two or three layers (2 or 3 ply).

199 The surface morphology of the inner, middle and outer layers of a 3 ply surgical mask is shown in

200 Fig. 2G, H and I, respectively.

201 Filtering efficiency

202 To find out if there is any correlation between surface structure and filtering efficiency, we 203 measured the filtering efficiency of selected cloth masks (CM3, CM7, CM9, CM18), and one 204 surgical mask (SM). The filtering efficiency is reported in Fig. 3A. The filtering efficiency was 205 measured by particle counting method using ambient particulate matter. The particle size 206 information is shown in Fig. 3B. Around 98\% particles in ambient air was smaller than $10 \mu \mathrm{m}$ 207 which is consistent with a recent study reported by our group (Rauniyaar, Aryal \& Neupane, 2019). 208 Thus, the filtering efficiency of face mask reported in Fig. 3A can be considered as PM $_{10}$ filtering 209 efficiency. Fig. 3A also shows that filtering efficiency of CMs ranged from $63 \%$ to $84 \%$ with 210 poorest efficiency of $63 \%$ measured for CM9. The filtering efficiency of surgical mask was found 211 to be $94 \%$.

212 Figure 3: Measurement of filtering efficiency. (A) Filtering efficiency of selected cloth face masks (CM) and a surgical mask (SM). The error bars represent standard deviation in each efficiency value reported $(n=3)$. (B) Size distribution of ambient particulate matter used in this study. 
219 Stretching effect on mask surface

220 We also explored how the surface of a mask changes on stretching the mask. To explore this,

221 surface of a cloth mask was stretched and microscopically observed the surface while being

222 stretched. Representative images of mask CM7 in stretched (change in length of mask/original

223 length of mask $=\Delta \mathrm{L} / \mathrm{L} \sim 0.05$ ) and un-stretched conditions is shown in Fig. 4A and B, respectively.

224 Careful comparison of these images shows that on stretching surface is distorted with increase in

225 pore size and change in shape. We found similar effects for other cloth masks although extent of

226 distortion was different. We did not find such changes for surgical mask.

227

228

229

230

231

232

233

234

235

236

237

238

239

Figure 4: Effect of stretching on mask surface. (A) Bright field microscopy images of

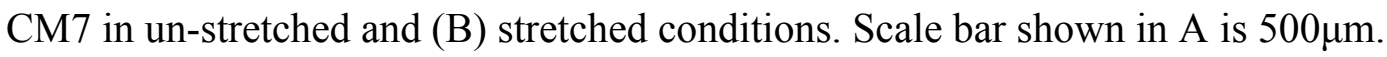

\section{Effect of washing and drying}

To explore the effect of washing and drying in the filtering efficiency, we selected a cloth mask CM9. The filtering efficiency measured after each washing and drying cycle for up to 4 cycles is shown in Fig. 5. With increase in washing and drying cycles there is gradual decrease in filtering efficiency $\left(\mathrm{R}^{2}=0.99\right)$.

Figure 5: Effect of washing and drying on filtering efficiency. Filtering efficiency for CM9 measured after washing and drying cycles. The error bars represent standard deviation in each efficiency value reported $(n=3)$ 
240 To find the possible cause of change in filtering efficiency with washing and drying we imaged

241 mask surface after each cycle. The representative images are shown in Fig. 6.

242

243

244

245

246

\section{DISCUSSION}

248

249

250

251

252

253

254

255

256

257

258

259

260

261

262
Figure 6. Optical images after washing and drying cycles. (A) Bright field microscopic images of unwashed CM9, and after (B) first, (C) second, (D) third, and (E) fourth washing and drying cycles. The scale bar shown in $\mathrm{A}$ is $500 \mu \mathrm{m}$ and applied to all images.

\section{Surface characterization}

It is very obvious from the images shown in Fig. 2A-F that pore size, shape, inter pore distance, and number of pores per field of view were very different for all the CMs. For examples, CM7 has the smallest and nearly circular pores and CM9 has largest pores and pores are nearly hexagonal, CM12 has medium sized triangular pores. If pores of all CMs are closely inspected, the pores are not perfectly clear but contain microfibers passing from one end of pore to next.

It is evident that the inner (Fig. 2G) and outer (Fig. 2I) layers of surgical face mask have very similar surface structure with distinctly visible interwoven cellulose fibers and open spaces. Although features in middle layers (Fig. 2H) are not distinctly discernible due to limited resolution of microscopic set up used in this study, we can tell that this layer has mocro/nanoporous membrane like structures. The presence of three layers, with middle layer having very small pores, tells that medical mask may be efficient in blocking the particulate matter.

We also explored if there is any difference on the interior surface of cloth masks and surgical mask. This was achieved by scanning the mask surface in axial direction while keeping the microscope objective fixed. Video was recorded during the axial scanning. A representative video collected 
263 for CM and SM is shown in supporting files S1 and S2, respectively. A careful comparison of two

264 videos tells that surgical mask has nicely interwoven microfibers with smaller pores indicating

265 better filtering efficiency in compassion to cloth masks.

\section{Filtering efficiency}

267 Filtering efficiency is particle size dependent (Belkin, 1997) with lower efficiency observed for 268 smaller sized particles. The size of particulate matter is source dependent and depending on source 269 size can be as small as few tenth of nanometer (Mirowsky et al., 2013). The size distribution of 270 ambient particles used in this work is shown in Fig. 3B. Lateral resolution $\left(D_{x y}\right)$ of microscope 271 system used in this study, based on the equation (2)(Hell, 2007; Stender et al., 2013; Neupane, 272 2016)

$$
D_{x y}=\frac{\lambda}{2 N A}
$$

with objective of 0.4 NA (NA=numerical aperture) and illumination light of wavelength $(\lambda)$ of 500 $\mathrm{nm}$, is around $0.6 \mu \mathrm{m}$. In this regard, actual size of particles measured can have some uncertainty. Nonetheless, classification of particles in three categories viz. $<5 \mu \mathrm{m}, 5-10 \mu \mathrm{m}$, and $>10 \mu \mathrm{m}$ was possible in our case. As shown in Fig. 3B, 98\% particles were smaller than $10 \mu \mathrm{m}$ which is in consistent with a recent study reported by our group(Rauniyaar, Aryal \& Neupane, 2019). Thus, the filtering efficiency of face mask reported in Fig. 3A can be considered as $\mathrm{PM}_{10}$ filtering efficiency.

Bright filed microscopy is frequently used in the determination of number density of micro-meter sized objects(Ricardo \& Phelan, 2008; Drey, Graber \& Bieschke, 2013; Rauniyaar, Aryal \& Neupane, 2019). The accuracy in such measurement is high if: 1) light scattering efficiency of an object to be imaged is high so they can be contrasted from the background, and 2) individual dispersion in the field of view can be achieved. In our case, scattering efficiency of particles is 
286 higher than background, and we maintained number of particles per field of view low so that

287 number density can be determined.

288 The filtering efficiency reported in Fig. 3A was negatively correlated with pore size of the masks, $289\left(\mathrm{R}^{2}=0.94\right)$ (Fig. 7). The poor efficiency of CM9 $(\sim 63 \%)$ is due to the presence of larger and open 290 pores (pores size $461 \pm 113 \mu \mathrm{m})$ and improved efficiency of CM7 $(\sim 84 \%)$ is due to presence of 291 smaller pores (pores size $100 \pm 53 \mu \mathrm{m}$ ). It is interesting to note that filtering efficiency of CM9 is 292 still $>60 \%$ although the pores are much bigger than the reported particle size. This contradiction 293 may be due to low pore density of the masks and mismatch of the pores in two layers during use. 294 The filtering efficiency of surgical mask was 94\%. Again, the excellent filtering efficiency can be

295

296

297 298 299 300 301

302 303

304 305 306 attributed to the presence of nicely interwoven microfibers with small pores (in middle layer) as reported in Fig. 2G-I. The difference in morphology of surgical and cloth masks is more obvious in supporting files S1 and S2 that show axial scanning of a cloth mask and a surgical mask, respectively.

Figure 7: Correlation between pore size and filtering efficiency. Each data point represents the mean pore size of mask (in micrometer) plotted as a function of filtering efficiency. The solid line is the linear fit to the data points.

We used a novel particle counting method to determine the filtering efficiency of face masks. Our method is passive sampling method as it measures the filtering efficiency of free falling dust particles without considering active suction to mimic human inhalation and exhalation. Efficiency was determined by the passive transfer of the particles from one side of the mask to the other. Therefore, this method provides the upper estimate of filtering efficiency or best-case-estimate of 
309

310

311

312

313

314

315

316

317

318

319

320

321

322

323

324

325

326

327

328

329

330

331

capture efficiency of mask. Although our method did not mimic the breathing condition, it allowed us to measure the filtering efficiency using ambient particulate matter. If a standard mask (N95 or 99) is available, our method could be a cheaper alternative to screen the relative efficiency of a mask of unknown efficiency and compare with a standard mask such as N95 or N99.

Filtering efficiency of cloth mask has also been reported in other studies by using active sampling method(Rengasamy, Eimer \& Shaffer, 2010; Shakya et al., 2017). They have reported that filtering efficiency of a cloth mask depends on the nature of particles (size and charge) used to measure the filtering efficiency, nature and design of mask. Their reported efficiency is in the range of 10-90\% and conclude that cloth mask perform poorer than N95 surgical mask. In contrast, we used passive method and ambient particulate matter. The surface characteristics of masks could also be different. Therefore, efficiency values are difficult to compare. Nonetheless, our conclusion is same i.e. cloth mask perform poorer than surgical masks.

\section{Stretching effect on mask surface}

Fig. 4 shows that mask (CM7) surface changed on stretching with significant increase in pore size in stretched mask. This observation is very important. People use cloth mask for months and the ear loops get stretched. The loop has to be knotted to make the mask fit better on the face. If mask having knotted ear-loop is used, it is very likely that whole mask surface gets stretched. Although, we did not measure the filtering efficiency while the mask is stretched, it can be easily inferred that efficiency will decline if mask having knotted ear-loop is used due to change in pore morphology as observed in Fig. 4. 


\section{Effect of washing and drying}

333 The filtering efficiency measured after each washing and drying cycle for up to 4 cycles is shown

334 in Fig. 5. We found a gradual decrease in filtering efficiency with an increase in washing and

335 drying cycle. As compared to unwashed mask of efficiency $\sim 63 \%$, after $4^{\text {th }}$ washing and drying

336 cycle there was $\sim 20 \%$ drop in filtering efficiency.

337 A close observation of images in Fig. 6 shows that small changes in surface morphology occur

338 after each cycle. The first change is increase in pore size and change in pore shape. The second

339 change is decrease in number of microfibers within the pore so that pore look more open i.e.

340 increase in pore clearance. These changes on the mask should be responsible for decline in

341 efficiency after washing and drying cycle, in consistent with the data reported in Fig. 5.

\section{CONCLUSIONS}

343 We studied the effect of surface morphology of locally available face masks on their particulate matter filtering efficiency. Filtering efficiency of cloth mask for ambient $\mathrm{PM}_{10}$ was poorer than surgical mask. The poor efficiency was due to the presence of larger sized pores. Our study also demonstrated that washing and drying cycle deteriorates the filtering efficiency due to change in pore shape and clearance. We also found that stretching of cloth mask surface alters the pore size and potentially decrease the filtering efficiency. Findings of this study suggest that cloth mask are not effective and effectiveness deteriorates if used after washing and drying cycles and used under stretched condition. We believe that findings of this study will be helpful to increase public awareness among populations of developing countries where such masks are very common and policy makers to make and implement basic guidelines for face masks for public use.

\section{SUPPORTING FILES}


355 S1. Axial scanning for a cloth mask. A short video that shows scanning of a cloth mask (CM9)

356 at various axial positions.

357 S2. Axial scanning for a surgical mask. A short video that shows scanning of surgical mask (SM)

358 at various axial positions.

\section{ACKNOWLEDGEMENTS}

363

364

365

366

We acknowledge laboratory facilities at Central Department of Chemistry, Tribhuvan University and Kathmandu Institute of Applied sciences.

\section{REFERENCES}

Belkin NL. 1997. The evolution of the surgical mask: filtering efficiency versus effectiveness. Infection Control \& Hospital Epidemiology 18:49-57.

Cherrie JW, Apsley A, Cowie H, Steinle S, Mueller W, Lin C, Horwell CJ, Sleeuwenhoek A, Loh M. 2018. Effectiveness of face masks used to protect Beijing residents against particulate air pollution. Occup Environ Med 75:446-452.

Chughtai AA, Seale H, MacIntyre CR. 2013. Use of cloth masks in the practice of infection control—evidence and policy gaps. Int J Infect Control 9:1-12.

Colbeck I, Nasir ZA, Ali Z. 2010. The state of indoor air quality in Pakistan - a review. Environmental Science and Pollution Research 17:1187-1196. DOI: 10.1007/s11356010-0293-3. 
377 Drey LL, Graber MC, Bieschke J. 2013. Counting unstained, confluent cells by modified bright378 field microscopy. Biotechniques 55:28-33.

379 Gurung A, Bell ML. 2012. Exposure to airborne particulate matter in Kathmandu Valley, Nepal. $380 \quad$ Journal of Exposure Science and Environmental Epidemiology 22:235.

381

382

383

384 385 386

Hell SW. 2007. Far-field optical nanoscopy. Science (New York, N.Y.) 316:1153-1158. DOI: 10.1126/science. 1137395 .

IQ Air. 2018. 2018 World Air Quality Report: Regional and City PM2.5 Ranking.

Kim K-H, Kabir E, Kabir S. 2015. A review on the human health impact of airborne particulate matter. Environment International 74:136-143. DOI: 10.1016/j.envint.2014.10.005.

MacIntyre CR, Chughtai AA. 2015. Facemasks for the prevention of infection in healthcare and community settings. Bmj 350:h694.

MacIntyre CR, Seale H, Dung TC, Hien NT, Nga PT, Chughtai AA, Rahman B, Dwyer DE, Wang Q. 2015. A cluster randomised trial of cloth masks compared with medical masks in healthcare workers. BMJ open 5:e006577.

Maleki H, Sorooshian A, Goudarzi G, Nikfal A, Baneshi MM. 2016. Temporal profile of PM10 and associated health effects in one of the most polluted cities of the world (Ahvaz, Iran) between 2009 and 2014. Aeolian Research 22:135-140. DOI: 10.1016/j.aeolia.2016.08.006.

Maxted B. 2011. Dust masks for Indian quarry workers: A comparative analysis of the filtering efficiency of fabrics. J. Humanitarian Eng 1:15-20.

Mirowsky J, Hickey C, Horton L, Blaustein M, Galdanes K, Peltier RE, Chillrud S, Chen LC, Ross J, Nadas A. 2013. The effect of particle size, location and season on the toxicity of urban and rural particulate matter. Inhalation toxicology 25:747-757. 
400 Mueller W, Horwell CJ, Apsley A, Steinle S, McPherson S, Cherrie JW, Galea KS. 2018. The

401

402

403

404

405

406

407

408

409

410

411

412

413

414

415

416

417

418

419

420

421 effectiveness of respiratory protection worn by communities to protect from volcanic ash inhalation. Part I: Filtration efficiency tests. International journal of hygiene and environmental health 221:967-976.

Neupane BB. 2016. Stimulated Emission Depletion Microscopy Resolves Nanoparticle Assembly on a Porous Membrane Surface. Nepal Journal of Science and Technology 17.

Rauniyaar J, Aryal GM, Neupane BB. 2019. Morphological study on particulate matter of Kathmandu valley. BIBECHANA 16:41-46.

Reche C, Moreno T, Amato F, Viana M, Van Drooge BL, Chuang H-C, Bérubé K, Jones T, Alastuey A, Querol X. 2012. A multidisciplinary approach to characterise exposure risk and toxicological effects of PM10 and PM2. 5 samples in urban environments. Ecotoxicology and environmental safety 78:327-335.

Rengasamy S, Eimer B, Shaffer RE. 2010. Simple respiratory protection-evaluation of the filtration performance of cloth masks and common fabric materials against 20-1000 nm size particles. Annals of occupational hygiene 54:789-798.

Ricardo R, Phelan K. 2008. Counting and determining the viability of cultured cells. Journal of Visualized Experiments: JoVE. DOI: 10.3791/752.

van der Sande M, Teunis P, Sabel R. 2008. Professional and home-made face masks reduce exposure to respiratory infections among the general population. PLoS One 3:e2618.

Shakya KM, Noyes A, Kallin R, Peltier RE. 2017. Evaluating the efficacy of cloth facemasks in reducing particulate matter exposure. Journal of Exposure Science \& Environmental Epidemiology 27:352-357. DOI: 10.1038/jes.2016.42. 
422 Sharma SK, Mandal TK, Saxena M, Sharma A, Datta A, Saud T. 2014. Variation of OC, EC,

423

424

425

426

427

428

429

430

431

432

433

434

435

436

WSIC and trace metals of PM10 in Delhi, India. Journal of Atmospheric and SolarTerrestrial Physics 113:10-22.

Singh MP, Singh VK, Patel DK, Tandon PK, Gaur JS, Behari JR, Yadav S. 2010. Face mask application as a tool to diminish the particulate matter mediated heavy metal exposure among citizens of Lucknow, India. Science of the total environment 408:5723-5728.

Stender AS, Marchuk K, Liu C, Sander S, Meyer MW, Smith EA, Neupane B, Wang G, Li J, Cheng J-X. 2013. Single cell optical imaging and spectroscopy. Chemical reviews $113: 2469-2527$.

Wang H, Zhuang Y, Wang Y, Sun Y, Yuan H, Zhuang G, Hao Z. 2008. Long-term monitoring and source apportionment of PM2. 5/PM10 in Beijing, China. Journal of Environmental Sciences 20:1323-1327.

World Health Organization. 2016. Ambient air pollution: A global assessment of exposure and burden of disease. 


\section{Figure 1}

Types of mask and survey.

(A) Images of some of the most commonly used cloth masks, and less commonly used

surgical mask (B). (C) Face mask use pattern in Kathmandu. The \% errors in the Fig. represent standard deviation. 
A
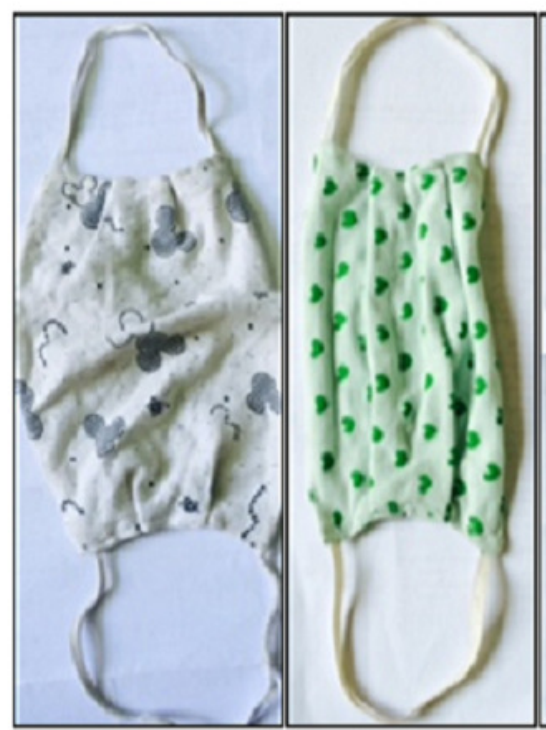

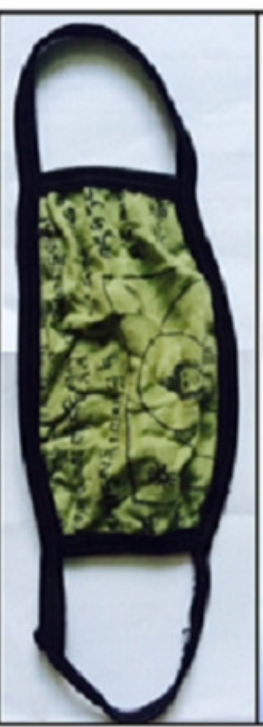

Cloth masks (CM)

B

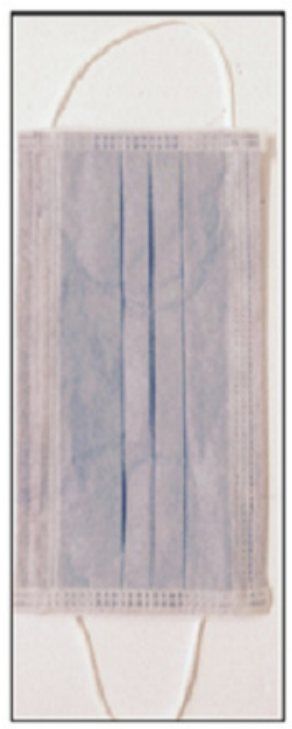

Paper/surgical mask (SM)

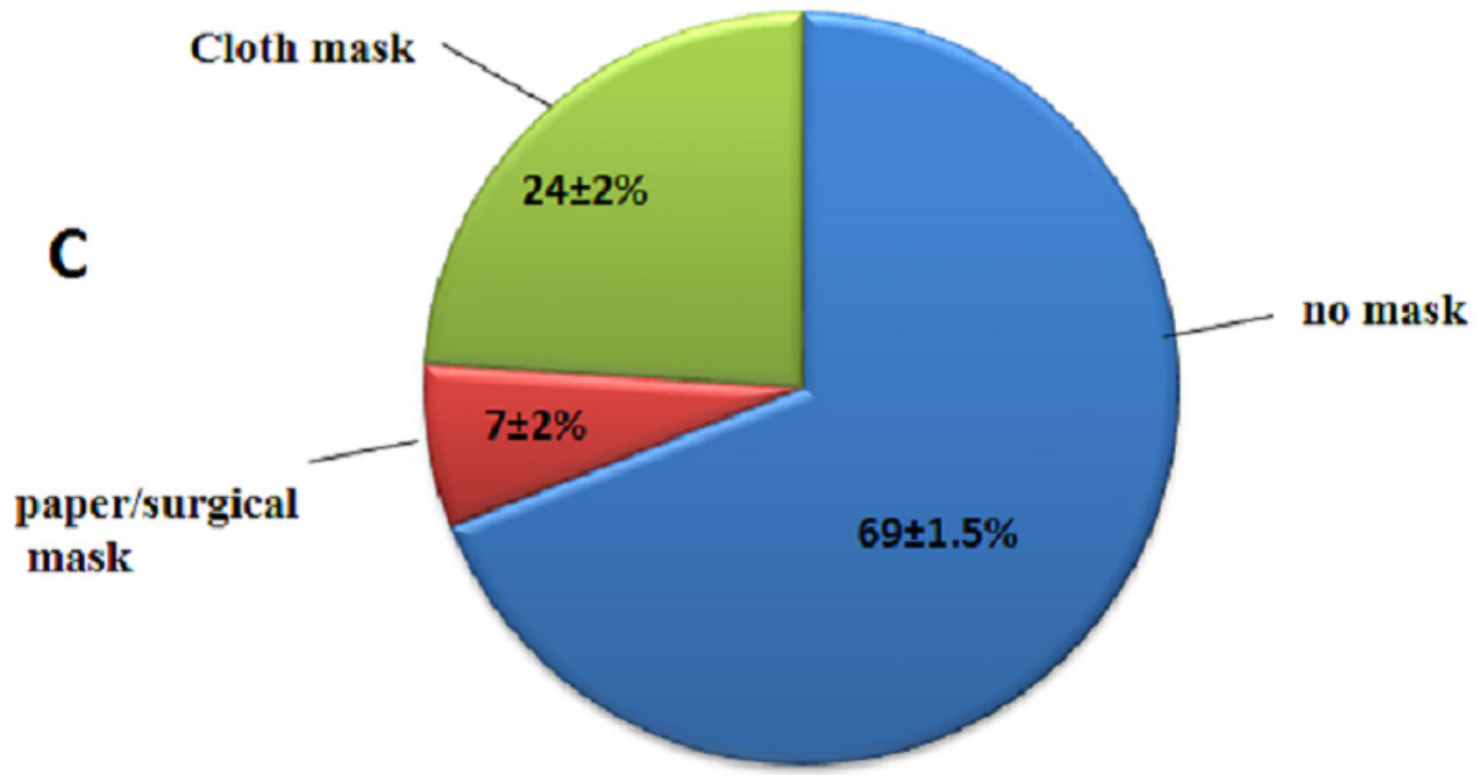


Figure 2

Bright field microscopic images of mask surfaces.

(A) Representative images for CM1, (B) CM3, (C) CM7, (D) CM9, (E) CM12, and (F) CM18. (G) Representative images of inner, $(\mathrm{H})$ middle, and $(\mathrm{I})$ outer layers of a three layered surgical mask. Scale bar shown A is $500 \mu m$ and applied to all images.
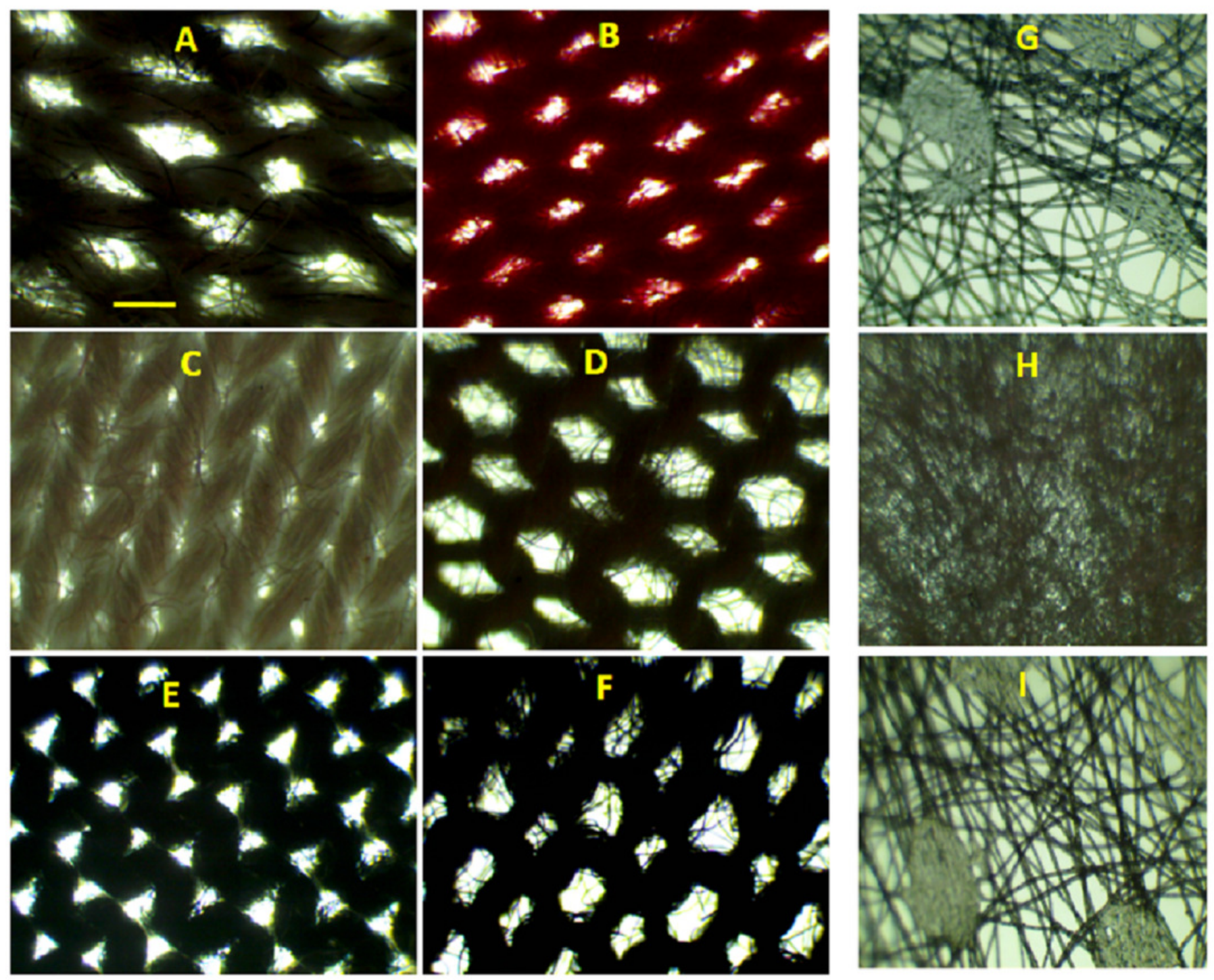
Figure 3

Measurement of filtering efficiency.

(A) Filtering efficiency of selected cloth face masks (CM) and a surgical mask (SM). The error bars represent standard deviation in each efficiency value reported $(n=3)$. (B) Size distribution of ambient particulate matter used in this study. 

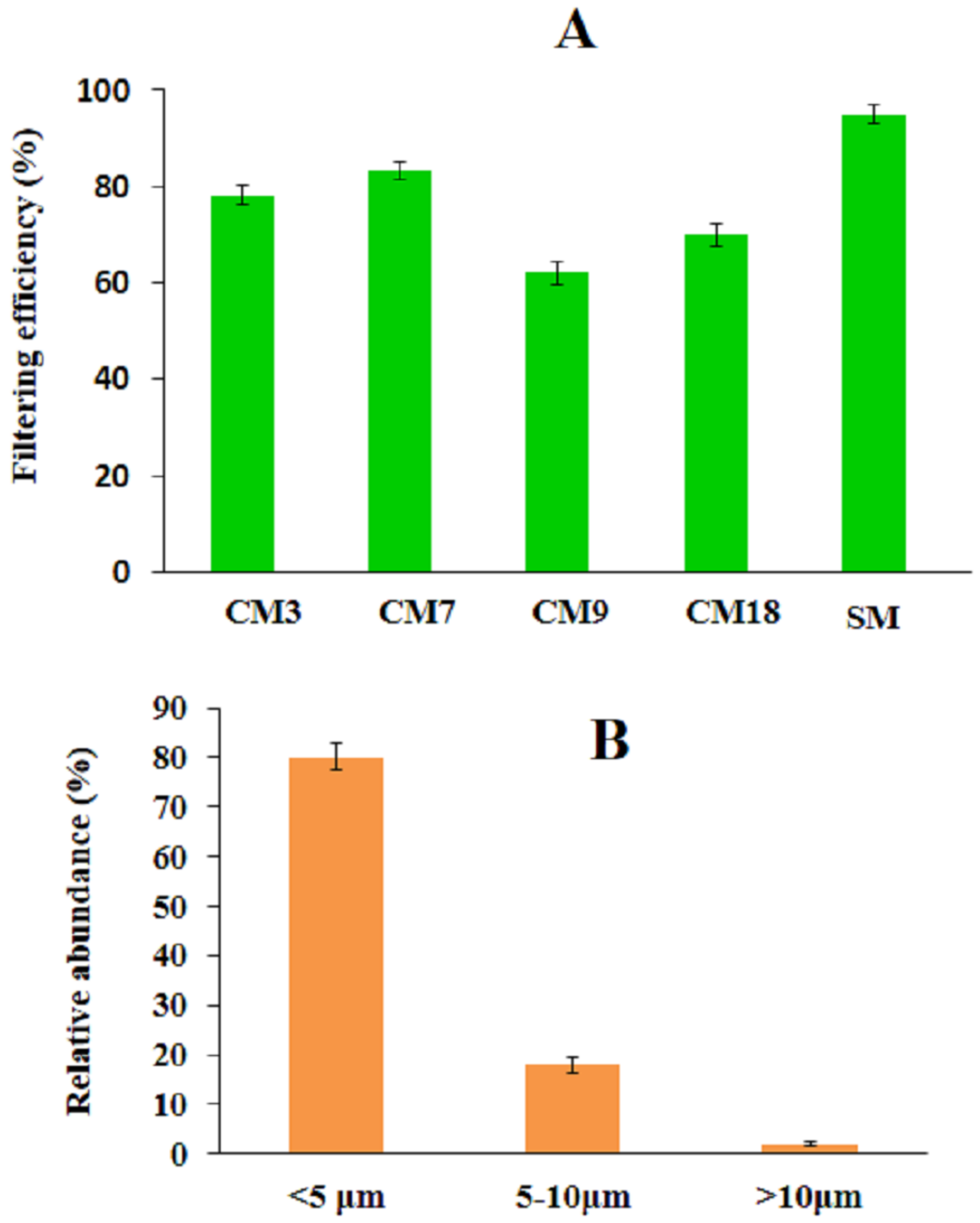

Particle size $(\mu \mathrm{m})$ 


\section{Figure 4}

Effect of stretching on mask surface.

(A) Bright field microscopy images of CM7 in un-stretched and (B) stretched conditions. Scale bar shown in $\mathrm{A}$ is $500 \mu \mathrm{m}$.
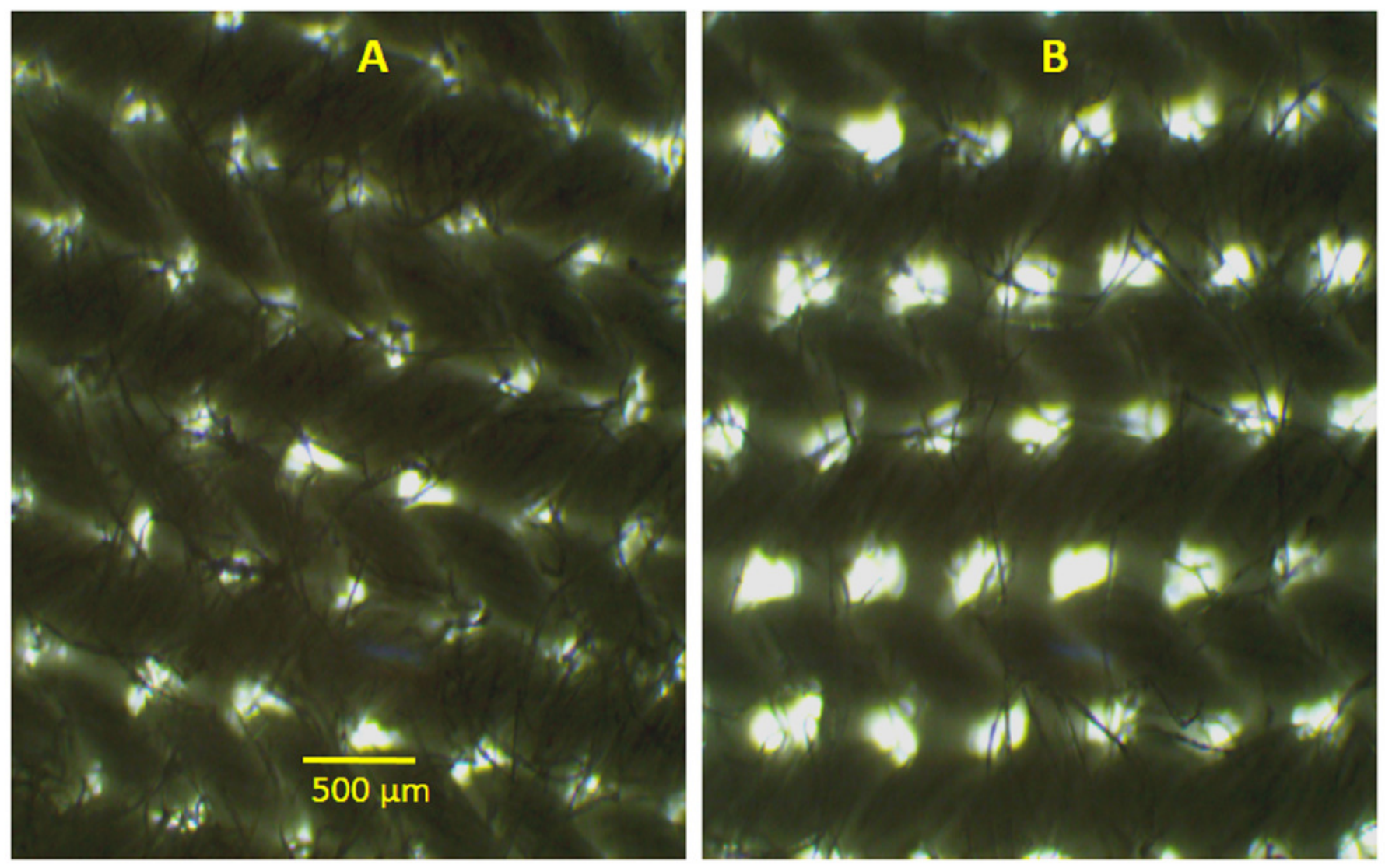
Figure 5

Effect of washing and drying on filtering efficiency.

Filtering efficiency for CM9 measured after washing and drying cycles. The error bars represent standard deviation in each efficiency value reported $(n=3)$

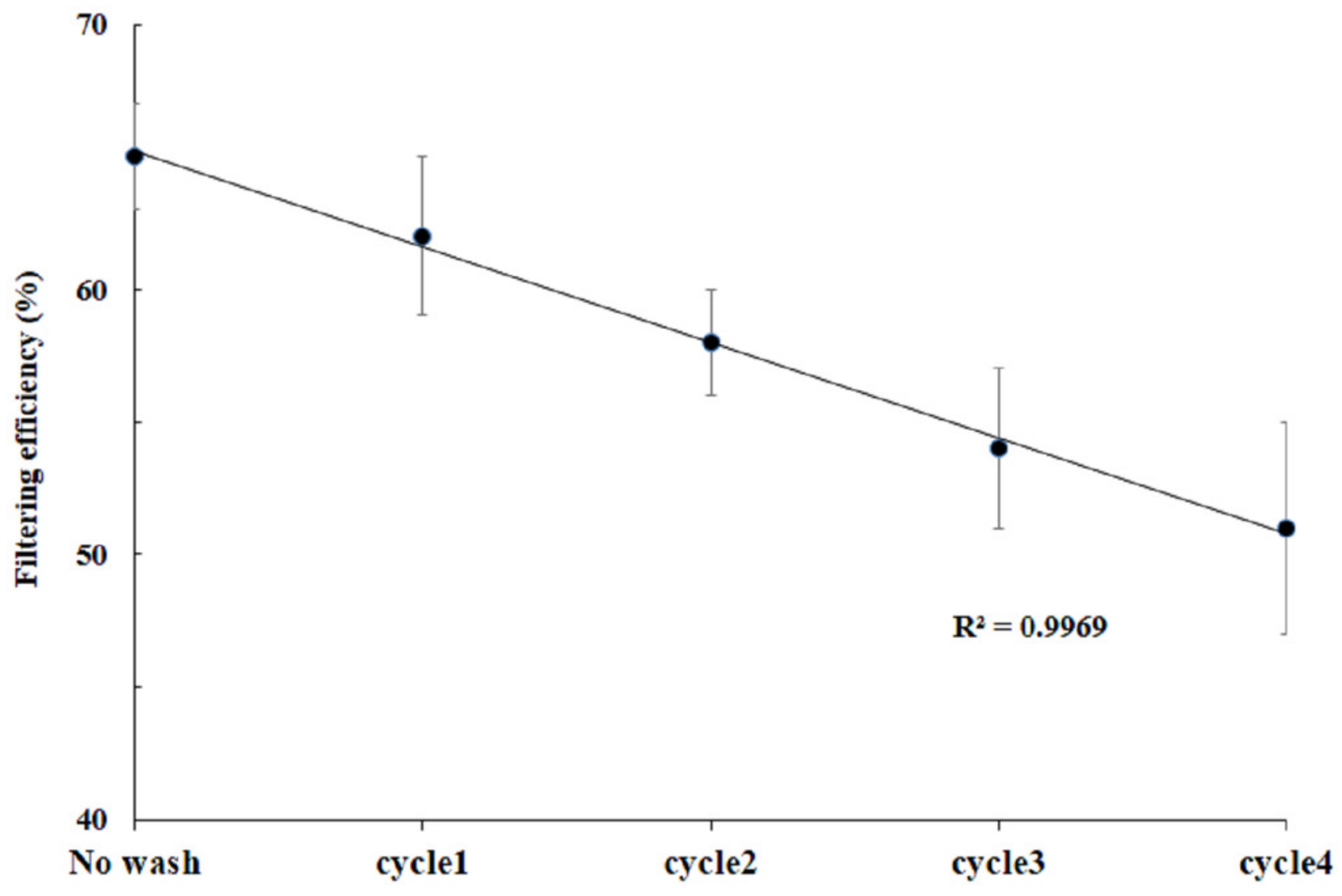




\section{Figure 6}

Optical images after washing and drying cycles.

(A) Bright field microscopic images of unwashed CM9, and after (B) first, (C) second, (D) third, and (E) fourth washing and drying cycles. The scale bar shown in $A$ is $500 \mu m$ and applied to all images.
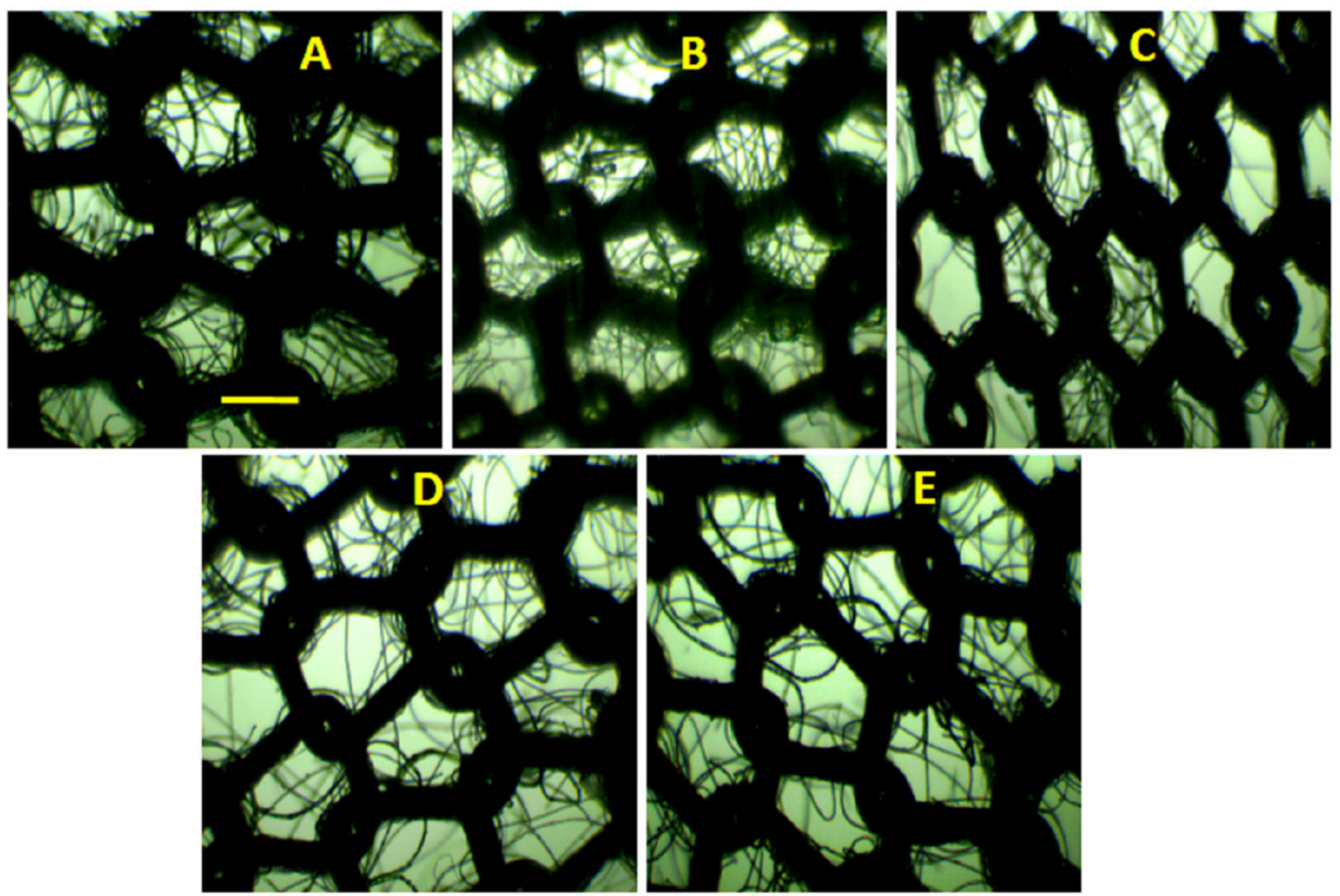
Figure 7

Correlation between pore size and filtering efficiency.

Each data point represents the mean pore size of mask (in micrometer) plotted as a function of filtering efficiency. The solid line is the linear fit to the data points.

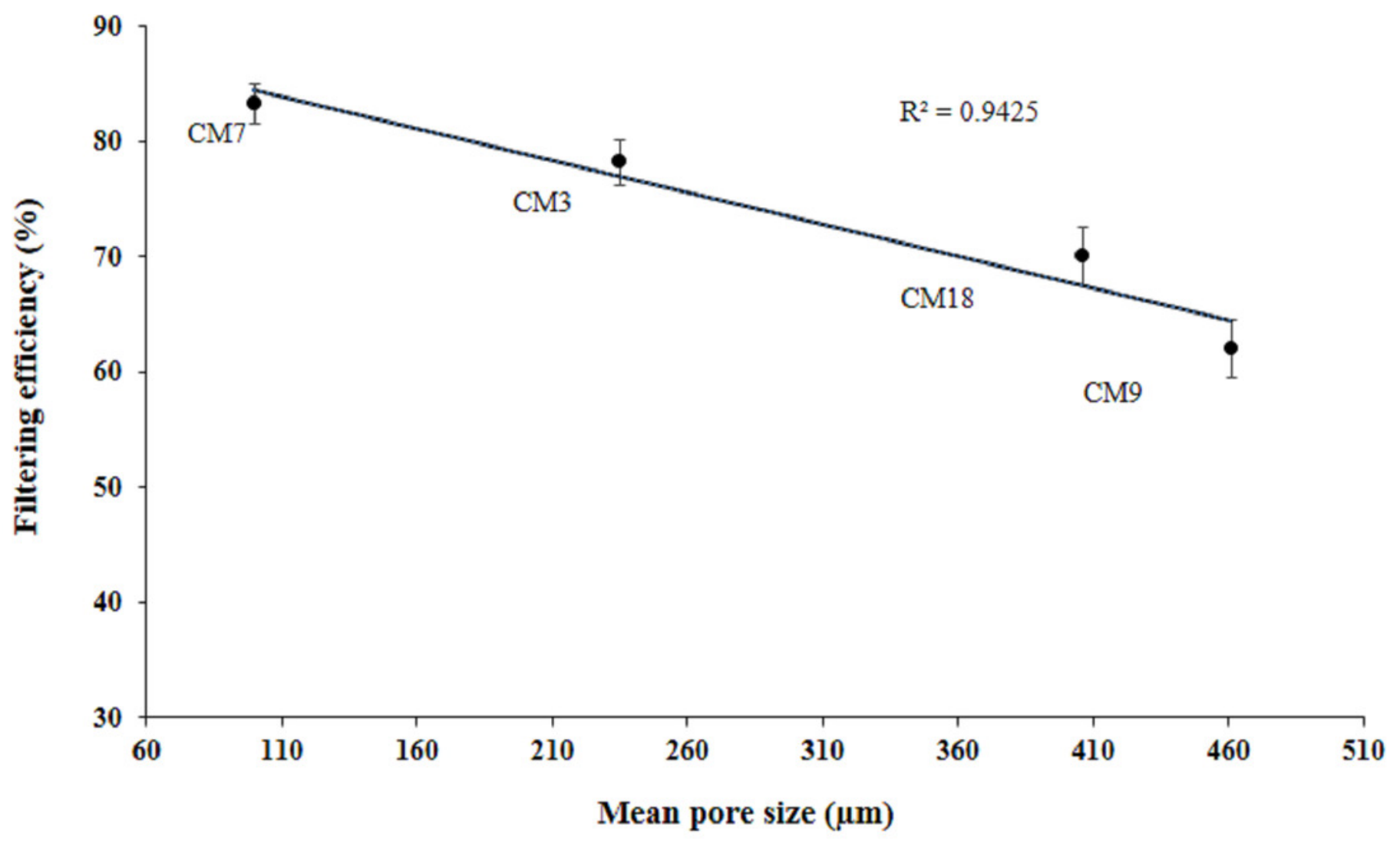

